\title{
Evaluation of Motor Sensory Blockade and Duration of Analgesia in Dexmedetomidine and Dexamethasone as Adjuvant to Bupivacaine
}

\author{
Ravi Gurbani ${ }^{1}$, Rashim Vachhani ${ }^{2}$ \\ ${ }^{1}$ Professor, Department of Anesthesiology, Chandulal chandrakar medical college, Durg, Chhattisgarh, India, ${ }^{2}$ Associate Professor, Department of \\ Anesthesiology Chandulal chandrakar medical college, Durg, Chhattisgarh, India
}

\section{Abstract}

Background: The studies are scant about the analgesic efficacy of the Dexamethasone and dexmedetomidine. Hence this study was taken up to assess the efficacy of Dexamethasone as an analgesic especially for upper limb surgeries. Subjects and Methods: 200 patients belonging to ASAI and ASAII were included in the study scheduled for upper limb surgeries after taking informed consent. These patients were divided in to two groups having 50 patients in each group. Group A received $20 \mathrm{ml}$ of $2 \%$ lignocaine with adrenaline plus $18 \mathrm{ml}$ of $0.5 \%$ bupivacaine plus $50 \mu \mathrm{g}$ of dexmedetomidine and group B received $20 \mathrm{ml}$ of $2 \%$ lignocaine with adrenaline plus $18 \mathrm{ml}$ of $0.5 \%$ bupivacaine plus $8 \mathrm{mg}$ of dexamethasone. Onset of sensory and motor block, duration of block, quality of intraoperative analgesia and duration of analgesia were recorded. Results: Our study revealed similar onset of sensory block in group A and B. Group A showed early onset and longer duration of motor block compared to group B. Intraoperative haemodynamics were similar in both groups. Conclusion: Our study concludes that using dexmedetomidine as adjuvant prolongs the duration of block and postoperative analgesia compared to dexamethasone with minimal or negligible adverse events.

Keywords: Bupivacaine, Dexmedetomidine, Dexamethasone.

Corresponding Author: Dr Rashim Vachhani, Department of Anesthesiology, Chandulal chandrakar medical college, Durg, Chhattisgarh, India.

Received: November 2019

Accepted: November 2019

\section{Introduction}

Pain is defined by the international association for study of pain as an "unpleasant sensory and emotional experience associated with actual or potential tissue damage or described in terms of such damage. Pain perception actually begins before birth. ${ }^{[1]}$ Surgical pain not only causes immediate nociceptive response but also results in changes in nociceptive activation pathways leading to hypersensitivity, hyperalgesia and allodynia. ${ }^{[2]}$

Brachial plexus block is a popular approach for upper limb surgeries as an alternative to general anesthesia. This type of anesthesia mainly helps in to achieve ideal operating conditions by producing muscular relaxation, maintaining stable intraoperative hemodynamic condition and sympathetic block which reduces postoperative pain, vasospasm and edema. ${ }^{[3]}$

Bupivacaine one is of the local anesthetic used most frequently as it has a longer duration of action varying from 3 to 8 hours. However, it has limiting factors like delayed onset, patchy or incomplete analgesia. ${ }^{[4]}$ To minimize these drawbacks many drugs like Neostigmine, Opioids, Haluronidase, Midazolam, Clonidine, Dexamethasone etc., have been added to local anesthetics to improve the quality and duration of action and postoperative analgesia. ${ }^{[5]}$
Among the $\alpha 2$ agonists clonidine and dexmedetomidine are commonly used. Dexmedetomidine is a highly selective $\alpha 2$ agonist with sedative and analgesic properties with minimal respiratory depression. It has a $\alpha 2 / \alpha 1$ selectivity ratio of (1600:1) which is eight times more potent than clonidine (200:1). It is shorter acting drug than clonidine with a distribution half- life of $9 \mathrm{~min}$ and elimination half- life of 2 hours. $^{[6,7]}$

However the studies are scant about the analgesic efficacy of the Dexamethasone and dexmedetomidine. Hence this study was taken up to assess the efficacy of Dexamethasone as an analgesic especially for upper limb surgeries.

\section{Subjects and Methods}

A randomized single blinded study was taken up among 200 patients aged between 18 to 70 years undergoing upper limb surgeries in Hospitals attached to Medical College. Ethical clearance was obtained before Institutional Ethical review committee. An informed written consent was obtained from all the patients. The inclusion and exclusion criteria were,

\section{Inclusion Criteria:}

Patients with ASA class I and II and Patients aged between 18 to 70 years. 


\section{Exclusion criteria:}

Exclusion criteria were patients with a history of significant neurological, psychiatric, neuromuscular, cardiovascular, pulmonary, renal, hepatic disease; alcoholism or drug abuse; pregnancy or lactating women; and patients receiving adrenoceptor agonist or antagonist therapy or chronic analgesic therapy. Also excluded were patients with morbid obesity, diabetes, peripheral vascular disease, suspected coagulopathy, or known allergies.

Patients were randomly allocated in this double blind study (using a sealed envelope technique) into two groups. group A $(\mathrm{n}=50)$ Patients received $20 \mathrm{ml}$ of $2 \%$ lignocaine with adrenaline plus $18 \mathrm{ml}$ of $0.5 \%$ bupivacaine plus $50 \mu \mathrm{g}$ of dexmedetomidine $(0.5 \mathrm{ml}$ drug plus $1.5 \mathrm{ml} \mathrm{NS})$, a total volume of $40 \mathrm{ml}$. Group B $(\mathrm{n}=50)$ Patients received $20 \mathrm{ml}$ of $2 \%$ lignocaine with adrenaline plus $18 \mathrm{ml}$ of $0.5 \%$ bupivacaine plus $8 \mathrm{mg}$ of dexamethasone $(2 \mathrm{ml})$, a total volume of $40 \mathrm{ml}$. The anesthesiologist performing the block and observing the patient was blinded to the treatment group. Data collection was done by the same anesthesiologist who was unaware of the group allocation.

Patient was taken to OT after starting ringer lactate infusion using $18 \mathrm{G} \mathrm{I}$.V cannula in the non - operated hand. Baseline values of heart rate, ECG, non-invasive blood pressure, peripheral oxygen saturation, respiratory rate was noted before execution of block technique. The study drug was prepared by an anaesthesiologist who was not involved in the study. Patient was asked to lie supine and head of the patient was turned to the contralateral side. Interscalene groove was identified and the site was cleaned with povidone iodine solution.

A superficial skin wheal was made one finger breadth above clavicle in the interscalene groove with $0.5 \%$ lignocaine. A $5 \mathrm{~cm}$ insulated nerve stimulator needle was attached to a nerve stimulator and the current to be delivered being set at $2.0 \mathrm{~mA}$ and a pulse width of $100 \mu \mathrm{s}$. Needle direction was almost perpendicular with slight inclination towards contralateral nipple and desired response in the form of muscle twitch of fingers were seeked. Once the desired response was attained, current was reduced to $0.5 \mathrm{~mA}$ and if the response still persisted, the drugs were injected after negative aspiration for blood before injecting the drugs in aliquots of $3 \mathrm{ml}$ to a total volume of $40 \mathrm{ml}$.

Onset of sensory block was assessed by spirit swab method. Assessment of motor block was done using the Bromage score.

Grade 0: Normal motor function with full flexion and extension of elbow, wrist, and fingers

Grade 1: Decreased motor strength with ability to move the fingers only

Grade 2: Complete motor block with inability to move the fingers

Surgery duration was noted. Side effects like dryness of mouth, nausea, vomiting and complications like LA toxicity, pneumothorax and post block neuropathy were monitored. Duration of sensory block was defined as the time interval between the end of drug administration and complete resolution of anaesthesia on all nerves.10 The duration of motor block was defined as the time interval between the end of drug administration and the recovery of complete motor function of hand and forearm.
The data was compiled and subjected to statistical analysis using Statistical Package for Social Sciences (SPSS), version 15. Demographic and hemodynamic data were subjected to Student's t-test and for statistical analysis of onset time and duration of sensory and motor blocks, and DOA unpaired ttest was applied. P-value $<0.05$ was considered as statistically significant and $\mathrm{P}<0.001$ as highly significant.

\section{Results}

Regarding the age and sex distribution, there was no difference among the two groups taken up for study. The youngest patient in dexmedetomidine group (Group A) was of 20 years whereas oldest was of 54 years. In dexamethasone group (Group B) the youngest patient was of 22 years wheres oldest was of 60 years [Table1].

Table 1: Age distribution among the patients
\begin{tabular}{|l|l|l|}
\hline Age in years & Group A $(\mathbf{n}=\mathbf{1 0 0})$ & Group B $(\mathbf{n}=\mathbf{1 0 0})$ \\
\hline $18-30$ years & 26 & 24 \\
\hline $31-50$ years & 44 & 48 \\
\hline $41-70$ years & 30 & 28 \\
\hline
\end{tabular}

Table 2: Time for onset of sensory block

\begin{tabular}{|l|l|l|}
\hline Time for onset & Group A $(\mathbf{n}=\mathbf{1 0 0})$ & Group B $(\mathbf{n}=\mathbf{1 0 0})$ \\
\hline $3-6$ minutes & 58 & 48 \\
\hline $7-10$ minutes & 36 & 42 \\
\hline$>=11$ minutes & 6 & 10 \\
\hline Mean time & 5.4 minutes & 6.0 minutes \\
\hline
\end{tabular}

Table 3: Time for onset of motor block

\begin{tabular}{|l|l|l|}
\hline Time for onset & Group A $(\mathbf{n}=\mathbf{1 0 0})$ & Group B $(\mathbf{n}=\mathbf{1 0 0})$ \\
\hline$<=11$ minutes & 74 & 0 \\
\hline $12-15$ minutes & 26 & 18 \\
\hline$>=16$ minutes & 0 & 82 \\
\hline Mean time & 11.4 minutes & 18 minutes \\
\hline
\end{tabular}

Table 4: Duration of sensory block

\begin{tabular}{|l|l|l|}
\hline Time for onset & Group A $(\mathbf{n}=\mathbf{5 0})$ & Group B $(\mathbf{n}=\mathbf{5 0})$ \\
\hline$<=800$ minutes & 4 & 90 \\
\hline $801-900$ minutes & 38 & 6 \\
\hline$>=900$ minutes & 58 & 4 \\
\hline Mean time & 911 minutes & 730 minutes \\
\hline
\end{tabular}

Table 5: Duration of motor block

\begin{tabular}{|l|l|l|}
\hline Time for onset & Group A $(\mathbf{n}=\mathbf{5 0})$ & Group B $(\mathbf{n}=\mathbf{5 0})$ \\
\hline$<=700$ minutes & 0 & 84 \\
\hline $701-800$ minutes & 0 & 12 \\
\hline $801-900$ minutes & 54 & 0 \\
\hline$>=901$ minutes & 6 & 4 \\
\hline Mean time & 842 minutes & 613 minutes \\
\hline
\end{tabular}

The time taken for onset of sensory block was almost same in both groups (Table 2) whereas time taken for onset of motor block was much less when dexmedetomidine was used (Group D) as compared to Group X using dexamethasone [Table 2].

The time taken for onset of motor block was much lesser in group A using dexmedetomidine (mean time - 11.4 minutes) as compared to group B using dexamethasone (mean time 18 minutes) [Table 3].

Regarding the duration of sensory block, the block lasted much longer for dexemedetomidine group as compared to dexamethasone group [Table 4]. Similar results were 
obtained for duration of motor block where mean time for A group was much greater than B group [Table 5]. Regarding the onset of pain in the postoperative period, it was much later in patients given dexmedetomidine as compared to patients given dexamethasone

\section{Discussion}

Supraclavicular blocks are performed at the level of the brachial plexus trunks. Here, almost the entire sensory, motor and sympathetic innervations of the upper extremity are carried in just three nerve structures (trunks), confined to a very small surface area. 8 Consequently, typical features of this block include rapid onset, predictable and dense anesthesia along with its high success rate. Local anesthetics alone for supraclavicular brachial plexus block provide good operative conditions but have a shorter duration of postoperative analgesia.9 Hence various drugs such as opioids, clonidine, neostigmine, dexamethasone, midazolam, magnesium etc., were used as adjuvant with local anesthetics in brachial plexus block to achieve quick, dense and prolonged block, but the results are either inconclusive or associated with side-effects. ${ }^{[10]}$

We observed in our study that patients who underwent upper limb surgery after execution of supraclavicular BPB, addition of dexmedetomidine or dexamethasone to LA solution, shortens the motor block onset time and prolongs the duration of block time. BPB is one of the easiest, safest and most commonly performed peripheral nerve blocks in day to day practice of anaesthesia. Using adjuvants like dexmedetomidine or dexamethasone further enhances the onset, quality and duration of analgesia.

Dexamethasone as an adjuvant to local anesthetic for peripheral nerve or neuraxial block has various mechanisms of actions such as direct membrane action in unmyelinated fibers, vasoconstriction, action on potassium channels, and suppression of other inflammatory mediators. ${ }^{[11]}$ Though the exact mechanism of action has not been definitely elucidated, one or more of the above mechanisms alone or in combination could play a role in its use as an analgesic adjuvant. $^{[12]}$

Dexmedetomidine; a highly selective, $\alpha 2$-adrenergic agonist; has analgesic, sedative, anesthetic sparing effects when used in systemic route. ${ }^{[13]}$ Use of dexmedetomidine as an adjuvant mixed with local anesthetics has been performed with neuraxial anesthesia in both adult and pediatric patients. Mixing dexmedetomidine as adjuvant with local anesthetics during peripheral nerve and nerve plexus blockade has recently been practiced by anesthesiologists. ${ }^{[14]}$

\section{Conclusion}

The present study concludes that Dexmedetomidine is a better alternative for decreasing the onset of motor block along with enhanced quality and duration of supraclavicular block with safe profile. Dexmedetomidine and dexamethasone, both are good as adjuvants in peripheral nerve blocks.

\section{References}

1. Gorczyca R, Filip R, Walczak E: Psychological aspects of pain. Ann Agric Environ Med 2013, 1:23-7.

2. Ji R-R, Kohno T, Moore KA, Woolf CJ: Central sensitization and LTP: do pain and memory share similar mechanisms? Trends in neurosciences 2003, 26:696-705.

3. Hosalli V, Ganeshnavar A, Hulakund S, DS P: Comparison of dexmedetomidine and clonidine as an adjuvant to levobupivacaine in ultrasound guided axillary brachial plexus block: a randomised double blind prospective study. Int J Clin Diagn Res 2015, 3:1-7.

4. Pullerits J, Holzman RS: Pediatric neuraxial blockade. Journal of clinical anesthesia 1993, 5:342-54.

5. Muir WW, Hubbell JA: Handbook of Veterinary Anesthesia-E-Book: Elsevier Health Sciences, 2014.

6. Saadawy I, Boker A, Elshahawy M, Almazrooa A, Melibary S, Abdellatif A, Afifi W: Effect of dexmedetomidine on the characteristics of bupivacaine in a caudal block in pediatrics. Acta Anaesthesiologica Scandinavica 2009, 53:251-6.

7. Dahl V, Raeder J: Non $\square$ opioid postoperative analgesia. Acta anaesthesiologica scandinavica 2000, 44:1191-203.

8. Singh S, Aggarwal A: A randomized controlled double-blinded prospective study of the efficacy of clonidine added to bupivacaine as compared with bupivacaine alone used in supraclavicular brachial plexus block for upper limb surgeries. Indian journal of anaesthesia 2010, 54:552.

9. Das A, Majumdar S, Halder S, Chattopadhyay S, Pal S, Kundu R, Mandal SK, Chattopadhyay S: Effect of dexmedetomidine as adjuvant in ropivacaine-induced supraclavicular brachial plexus block: A prospective, double-blinded and randomized controlled study. Saudi journal of anaesthesia 2014, 8:S72.

10. Mukherjee K, Das A, Basunia SR, Dutta S, Mandal P, Mukherjee A: Evaluation of Magnesium as an adjuvant in Ropivacaine-induced supraclavicular brachial plexus block: A prospective, double-blinded randomized controlled study. Journal of research in pharmacy practice 2014, 3:123.

11. Lirk P, Hollmann MW, Strichartz G: The science of local anesthesia: Basic research, clinical application, and future directions. Anesthesia \& Analgesia 2018, 126:1381-92.

12. Parameswari A, Krishna B, Manickam A, Vakamudi M: Analgesic efficacy of dexamethasone as an adjuvant to caudal bupivacaine for infraumbilical surgeries in children: A prospective, randomized study. Journal of anaesthesiology, clinical pharmacology 2017, 33:509.

13. Kamibayashi T, Maze M: Clinical uses of $\alpha 2$-adrenergic agonists. Anesthesiology: The Journal of the American Society of Anesthesiologists 2000, 93:1345-9.

14. Brummett CM, Williams BA: Additives to local anesthetics for peripheral nerve blockade. International anesthesiology clinics 2011, 49:104.

Copyright: () the author(s), publisher. Academia Anesthesiologica International is an Official Publication of "Society for Health Care \& Research Development". It is an open-access article distributed under the terms of the Creative Commons Attribution Non-Commercial License, which permits unrestricted non-commercial use, distribution, and reproduction in any medium, provided the original work is properly cited.

How to cite this article: Gurbani R, Vachhani R. Evaluation of Motor Sensory Blockade and Duration of Analgesia in Dexmedetomidine and Dexamethasone as Adjuvant to Bupivacaine. Acad. Anesthesiol. Int. 2019;4(2):292-4.

DOI: dx.doi.org/10.21276/aan.2019.4.2.65

Source of Support: Nil, Conflict of Interest: None declared. 